\title{
Epiphora During the First Year of Life
}

\author{
C. J. MACEWEN and J. D. H. YOUNG \\ Dundee
}

\begin{abstract}
Summary
A cohort of 4,792 infants was observed in order to determine the incidence and natural history of epiphora during the first year of life. Evidence of defective lacrimal drainage was present in $964(20 \%)$ at some time during the year. $95 \%$ became symptomatic during the first month of life. Spontaneous remission occurred throughout the year and $96 \%$ had resolved before the age of one. This study provides no evidence to support probing before the age of one year.
\end{abstract}

Infants with epiphora are a common problem in clinical ophthalmology. It is generally accepted that the condition is the result of a congenital abnormality of the lacrimal drainage system, in the form of a membranous obstruction at the lower end of the naso-lacrimal duct (NLD). ${ }^{1}$ In addition it is recognised that there is a high rate of spontaneous resolution. ${ }^{2,3}$ However, despite it's frequency, relatively little is known about the incidence or natural history of epiphora in young children.

Published reports concerning the incidence of epiphora in infants provide widely different figures, ranging from $1.2 \%$ to $6 \% .^{4-7}$ The large variation reflects the different methodologies used and populations studied. For example, the lowest figure of $1.2 \%$ refers to an estimation of the incidence, based on numbers of children with the condition actually referred to hospital projected into the general population. ${ }^{4} \mathrm{~A}$ figure of $1.75 \%$ was derived from the incidence found in 1,538 infants attending the paediatric out-patient clinic, apparently without any follow-up. ${ }^{5}$ An incidence of $5 \%$ was reported by Cassady in 1948 , based on a general paediatric clinic population, but the ages of the children were not stated, nor the reasons for referral. ${ }^{6}$ Per- haps the most reliable estimate of the incidence is $6 \%$. This comes from a follow-up study of 200 consecutive, unselected newborn infants. $^{7}$

Information on the rate of spontaneous remission is also limited. The studies that are available were based on small clinic populations, referred for treatment of their epiphora and probing was usually undertaken in a number of cases before the end of the year. ${ }^{2,3}$ These series do quote high rates of spontaneous remission, up to $100 \%$ before the age of one, ${ }^{7}$ but the age of entry into these studies was unclear, and a variety of 'consecutive, medical' treatments were used.

Current advice on the management of NLD obstruction and on the timing of probing is based on unreliable data. We, therefore, considered there was the need to determine the incidence and natural history of epiphora in a large population of infants observed without treatment.

\section{Methods}

Tayside Health Board operates a very comprehensive computer-based paediatric surveillance programme. Every child born in Tayside is assessed at 8,20, and 37 weeks of 
age. At each visit the child is examined by either an experienced health visitor or a community paediatrician. The attendance rates are very high.

This surveillance system, which has now been in successful operation for a number of years, was utilised as a vehicle for our study. The study group comprised a cohort of all children born during 1988 who were resident in Tayside at the time of their first ( 8 week) screening. With the agreement of the community paediatricians and all local general practitioners (GPs), an additional form was included in the usual screening documents. This form required the paediatrician to ask the mother a series of simple standard questions, about the occurrence of any watering or stickiness from one or both eyes during the baby's lifetime. Appointments were made at the eye clinic for all children who were reported to have one or both eyes watering at either of the screening times. A self-assessment form was sent to the parents and/or GPs of patients who were unable to attend.

All children had at least two screenings by an experienced paediatric health worker. A 37 week screening form was also completed if either or both of the childs eyes had been symptomatic at the 20 weeks screening. Information collected from the forms included the ages of onset and remission of the epiphora.

In addition a letter was sent to all GPs in Tayside informing them of the study, and asking them to refer any children with watery or sticky eyes born during that year to the eye clinic, at the same time to avoid the use of topical antibiotics except in cases with conjunctival hyperaemia.

At the eye clinic, examination was particularly directed to the following points. External examination to confirm the presence or absence of the lacrimal puncta, lid position anomaly such as epiblepharon, or craniofacial anomaly. The cornea and conjunctiva were inspected. The fluorescein disappearance test (FDT) was carried out on all infants examined. This test has been shown to be very sensitive in the detection of lacrimal outflow anomalies. ${ }^{9.10}$ It was used in this study to provide valuable, objective confirmation of outflow obstruction to complement the parents observations. Lacrimal sac massage, topical antibiotics or other 'medical' treatments were not prescribed. Parents were advised to simply wipe the sticky lids and lashes with cooled boiled water.

\section{Results}

\section{Study Population}

The cohort consisted of all 4,792 children born between January 1, 1988 and December 31, 1988 and who were resident in Tayside at aged 8 weeks. During the first 12 months of follow-up 139 children either moved away from the area or were lost to follow-up and 10 died. Follow-up was complete on the remaining 4,643 children $(97 \%$ of the original cohort).

To study the natural history of the condition we evaluated each eye individually, in 73 cases the condition was bilateral. From the original group of 1,068 eyes which had been reported as being watery or sticky, 15 were in children subsequently lost to follow-up. There were 16 eyes with epiphora due to epiblepharon, (all had normal fluorescein disappearance tests) and another 14 eyes suffered isolated episodes of conjunctivitis with no preceding of subsequent history of epiphora or discharge. During the year three children with epiphora (four eyes) had anaesthetics for the management of unrelated disease. On ethical grounds probing was undertaken during the same anaesthetic. Therefore, the natural history of the condition was studied in the remaining 1,019 eyes.

\section{Incidence}

Of the original cohort of 4,792 children, 964 children had evidence of defective lacrimal drainage at some time during their first year of life. The incidence of this abnormality in a general population cohort was therefore $20 \%$ of all infants during the first year of life. By the age of one year 34 children ( 41 eyes) were still symptomatic $(0.7 \%)$

\section{Onset of Epiphora}

In $95 \%$ of cases the onset of epiphora or discharge occurred during the first month of life. A more detailed assessment of the first two months indicates that in 834 cases the epiphora had been observed during their first week of life, 90 in the second, 31 in the third 
AGE AT ONSET OF EPIPHORA

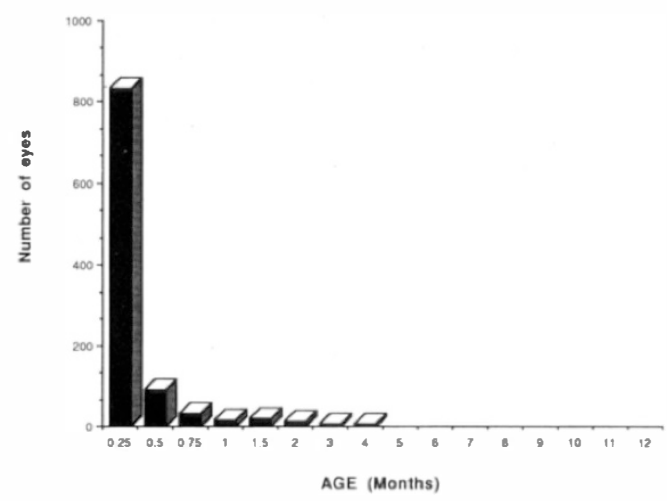

Fig. 1. Age at onset of epiphora. The first two months are broken down into smaller time intervals to demonstrate the very early onset in most cases. In $95 \%$ of eyes epiphora presented during the first month of life.

and a further 17 during the fourth week (Fig. 1). Another 34 started during the second month of life, seven during the third month and six in the fourth month.

\section{Spontaneous Resolution}

Spontaneous resolution was observed from the first month through to the end of the 11th month. Fig. 2 illustrates graphically the actual number that resolved during each month of life. Three hundred and sixty four eyes resolved during the first month, 219 in the second, 144 in the third, 69 in the fourth, 59 in the fifth, 50 in the sixth, 33 in the seventh, 17 in the eighth, 11 in the ninth, 10 in the tenth,

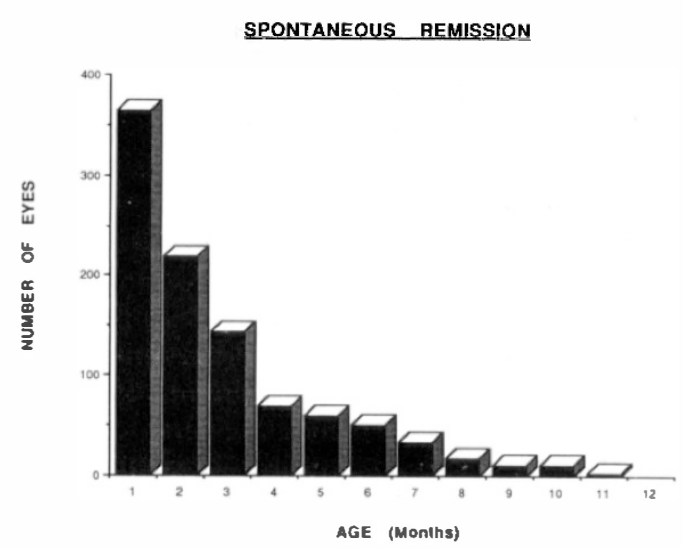

Fig. 2. Spontaneous remission. Each column represents the number of eyes that showed resolution of symptoms during that month of life. two in the 11th and none in the 12th month. The total number of eyes with epiphora at any one time therefore reduced steadily over the course of the year (Fig. 3). There were 834 symptomatic eyes during the first month of life, 608 in the second, 423 in the third, 286 in the fourth, 223 in the fifth, 164 in the sixth, 114 in the seventh, 81 in the eighth, 64 in the ninth, 53 in the tenth, 43 in the 11th and 41 in the last month of the year. The month by month resolution rate was approximately $30 \%$, until the end of the eighth month when it declined. The overall rate of spontaneous resolution by the end of the first year of life was $96 \%$.

\section{Probability of Spontaneous Resolution}

The probability of spontaneous remission by the end of the first year of life can be evaluated from these figures (Table I). This information may be useful when discussing the treatment possibilities with the parents of affected infants. For example, a child suffering from epiphora during the third month of life has a $90 \%$ chance that it will have resolved spontaneously by the age of one year and even as late as the ninth month, $36 \%$ will have improved before the child is one year old.

\section{Infection}

No child suffered from obstructed dacryocys-

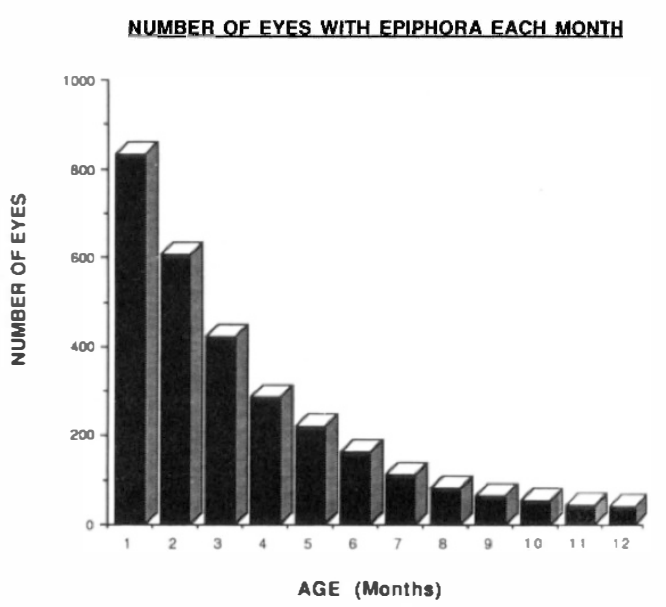

Fig. 3. Eyes With Epiphora during The First Year of Life. After exlusions, 4,643 infants were followed for the full 12 months. Each column represents that number. or eyes watering during that month. 
Table I Predicting Spontaneous Resolution. The table shows the percentage of infants at each month, who on follow-up, resolved before aged 12 months. These figures can be used to predict the chances of remission when discussing management with parents.

$\begin{array}{lr}1 \text { MONTH } & 96 \% \\ 2 \text { MONTHS } & 93 \% \\ 3 \text { MONTHS } & 90 \% \\ 4 \text { MONTHS } & 86 \% \\ 5 \text { MONTHS } & 82 \% \\ 6 \text { MONTHS } & 75 \% \\ 7 \text { MONTHS } & 64 \% \\ 8 \text { MONTHS } & 49 \% \\ 9 \text { MONTHS } & 36 \% \\ 10 \text { MONTHS } & 23 \% \\ 11 \text { MONTHS } & 5 \% \\ 12 \text { MONTHS } & 0 \%\end{array}$

titis, although there were ten children with mucocoeles. There were nine cases (18 eyes) in which conjunctivitis appeared as a superadded infection, secondary to defective lacrimal drainage. In all cases this settled with topical treatment. As previously noted, seven other patients (14 eyes) in the cohort developed conjunctivitis without any evidence of associated defect in lacrimal drainage.

\section{Discussion}

This report is the first large population-based study of the incidence and natural history of epiphora in infancy and has generated a number of significant findings. The follow-up data collection was $97 \%$ complete and we are therefore confident that our results are a true reflection of the incidence and natural history in the general population.

Firstly the incidence of $20 \%$ is much higher than that previously reported. The lower figures quoted in other studies were almost certainly due to methodological problems. The comprehensive paediatric screening available in Tayside has enabled us to ensure that all babies living in the area in 1988 were included in the survey from a very early age. This has not been a feature in other studies and the rapid improvement in the majority of cases during the first few months of life makes our higher figure more easily explainable.

The finding of such a high incidence of lacrimal drainage dysfunction with subsequent remission raises the possibility that this is not a pathological condition at all; simply a variation of normal development in infancy. Path- ological examination of the nasolacrimal ducts of stillborn infants has previously demonstrated imperforate membranes in $70 \%$ of the cases examined. ${ }^{1}$ There might also be a functional outflow delay as a consequence of immature lacrimal pump function. The early age of onset in the majority of those affected strongly supports the suggestion that tear secretion is present at birth.

The second important finding is the confirmation that spontaneous resolution of epiphora is very common and continues throughout the first year of life, as indeed previously suggested in smaller clinical studies. ${ }^{2.3}$ However, it has been suggested by several authors that if the condition is left untreated then this may lead to the complications of secondary infection and prolonged nasolacrimal duct impatency. ${ }^{4}$ In fact our secondary infection rate was remarkably low with only nine episodes of secondary conjunctivitis, all of which cleared quickly with the appropriate antibiotic treatment. The suggestion that untreated infants might develop lacrimal flow obstruction in later life seems unlikely, but cannot yet be refuted. Probing large numbers of infants who are, in any case, destined to be normal must in itself carry the potential risk of trauma induced lacrimal drainage obstruction.

Finally, the information contained in Table I can be usefully employed to indicate to parents the likelihood of spontaneous remission, making it easier for them to accept the delay in surgical intervention.

This study provides strong evidence to delay probing until at least 12 months of age. It, however, remains to be determined when the rate of spontaneous remission falls to below the point when treatment should be considered, thereby defining the optimum age to probe. Jointly with colleagues in other centres we are prospectively investigating the timing of probing for all children with epiphora after the age of one.

We are indebted to Sandra Murray, Barbara Hood and Margaret Davidson for administrative assistance. We would also like to thank Charles Guthrie and Stuart Pringle for statistical analysis.

\footnotetext{
References

${ }^{1}$ Cassady JV: Developmental anatomy of the naso-
} 
lacrimal duct. Arch. Ophthalmol 1952, 47: 141-58.

${ }^{2}$ Petersen RA and Robb RM: The course of congenital obstruction of the naso-lacrimal duct. $J$ Paediatr Strabism 1978, 15: 246-50.

${ }^{3}$ Paul TO: Medical management of congenital nasolacrimal duct obstruction. J Paediatr 1958, 22: 68-70.

${ }^{4}$ Ffookes OO: Dacryocystitis in infancy. $\mathrm{Br} J \mathrm{Oph}$ thalmol 1962, 46: 422-34.

${ }^{5}$ Stephenson S: A Preliminary communication on the affections of the tear passages in newly born infants. M Press and Circ 1899, 119: 103-4.
${ }^{6}$ Cassady JV: Dacryocystitis in infancy. Am J Ophthalmol 1948, 31: 773-80.

${ }^{7}$ Guerry D and Kendig EL: Congenital impatency on the naso-lacrimal duct. Arch Ophthalmol 1948, 39: 193-204.

${ }^{8}$ Patrick RK: lacrimal secretion in full term babies and premature babies. Trans Ophthalmol Soc UK 1974, 94: 283-90.

${ }^{9}$ Zappia RJ and Milder B: Lacrimal drainage function; 2. The fluorescein dye disappearance test. Am J Ophthalmol 1972, 74: 160-2.

${ }^{\text {II }}$ Katowitz JA and Welsh MG: Timing of initial probing and irrigation in congenital nasolacrimal duct obstruction. Ophthalmol 1987, 94: 698-705. 